\title{
The value of detecting pepsinogen and gastrin-17 levels in serum for pre-cancerous lesion screening in gastric cancer
}

\author{
G. YU, G. X. WANG*, H. G. WANG, F. F. MO, B. B. TANG \\ Department of Gastroenterology, Taizhou Municipal Hospital, Taizhou, Zhejiang, China \\ ${ }^{*}$ Correspondence: guoxiang19660422@163.com
}

Received August 25, 2018 / Accepted February 20, 2019

\begin{abstract}
The aim of the study was to estimate the value of detecting pepsinogen (PG) I, PGII and gastrin-17 (G-17) levels in serum for chronic atrophic gastritis (CAG) screening and to determine the clinical applicability of combined measurement of serum G-17, pepsinogens (PGI, PGII) and PGI/PGII ratio (PGR) as a screening test for CAG. The PGI, PGII and G-17 levels were detected by ELISA in 68 patients with CAG and 86 healthy volunteers who underwent gastroscopy for gastroduodenal diseases at Taizhou Municipal Hospital between January 2016 and December 2016. Concentrations of all measured serum markers were lower in patients with CAG in comparison to healthy volunteers and achieved statistical significance $(\mathrm{p}<0.01)$ in PGI (93.25 vs. 126.98) and PGR (12.67 vs. 17.09). Receiver operating characteristic (ROC) curve analysis revealed the optimal cut-off values for PGI, PGII, PGR and G-17 at $98.10 \mu \mathrm{g} / \mathrm{l}, 6.92 \mathrm{ng} / \mathrm{l}, 15.77$ and $1.94 \mathrm{pmol} / \mathrm{l}$, with sensitivities of $72.10 \%, 58.10 \%, 61.60 \%$ and $59.30 \%$, and specificities of $61.8 \%, 51.50 \%, 77.90 \%$ and $55.90 \%$, respectively. The areas under the curve (AUCs) of PGI, PGR and G-17 were $0.728,0.726$ and 0.556 , respectively. The increase of AUC was observed only in PGR and G-17 combination (0.741) with increased sensitivity (69.10\% vs. 61.60\%) of screening for CAG, whereas the specificity was reduced $(72.10 \%$ vs. $77.90 \%)$ in comparison to PGR alone. Combination of serum indicators can raise the diagnostic accuracy of CAG in some respects. However, further research including a larger sample size is necessary in order to accurately determine the sensitivity and specificity of combined detection of serum indicators.
\end{abstract}

Key words: pepsinogen, gastrin-17, chronic atrophic gastritis, precancerous lesions, gastric cancer

Chronic atrophic gastritis (CAG) is a well-established precursor lesion. The prevalence of CAG is higher in populations with higher rates of gastric cancer and the risk of gastric cancer is substantially increased in individuals with CAG $[1,2]$. Gastric cancer is the fourth most common malignant tumor worldwide and it is highly related to lifestyle, such as diet, alcohol consumption, smoking, and so on [3]. It is also the second most common cause of death from cancer. The high mortality and poor prognosis related to gastric cancer are the main causes of cancer death [4]. Early detection, early diagnosis and early treatment as a secondary prevention strategy for gastric cancer are key factors for reducing the mortality associated with gastric cancer and for prolonging survival among patients with gastric cancer. Therefore, it is particularly important to predict and screen high-risk groups for gastric cancer. CAG is a common precancerous lesion of gastric cancer [5-6] that needs regular follow-up to improve the early diagnosis rate and reduce the mortality associated with gastric cancer [7]. Furthermore, early diagnosis and treatment of CAG is important for preventing the occurrence of gastric cancer.

Currently, CAG and GC still need to be confirmed by pathological examination of specimens obtained by gastroscopy. However, the cost of gastroscopic screening is very high and the compliance of patients is poor. Thus, it is difficult to apply gastroscopy and biopsy for large-scale screening of CAG and GC. Fortunately, the serum concentrations of some specific molecules secreted by the gastric mucosa, such as pepsinogen (PG) and gastrin-17 (G-17) change when gastric mucosa atrophy occurs; thus, these molecules may serve as candidate markers for the screening of GC and CAG. Previous studies have reported that PG and G-17 could be used to monitor changes in gastric mucosal atrophy [8-9]. The purpose of this study was to estimate the value of detecting the levels of PG and G-17 in serum for screening of CAG and to determine the clinical applicability of combined measurement of serum G-17 and PGI, PGII and the PGI/ PGII ratio as a screening test for CAG. 


\section{Patients and methods}

Research population. This study is a case-control study. The subjects were patients who underwent gastroscopy for gastroduodenal diseases at Taizhou Municipal Hospital between January 2016 and December 2016. All subjects underwent gastroscopy before serological examination. Two biopsy specimens were obtained from the gastric antrum and gastric body. Furthermore, biopsy specimens were obtained when mucosal lesions were found on gastroscopy. The patients were divided into two groups according to the histopathological examination results: (1) Group CAG $(n=68)$ : gastric mucosa biopsy showed mild-to-moderate chronic atrophic gastritis with or without intestinal metaplasia, (2) Control group $(n=86)$ : gastric mucosa biopsy showed normal or mild-to-moderate non-atrophic gastritis in gastric mucosa. Distribution of sex and age within the two groups is shown in Table 1. No subject had a history of administering special medication (including proton pump inhibitors, H2 receptor antagonists, etc.) one week before inclusion into the study. The written consents were obtained from all subjects.

Serological detection. Blood samples were collected under fasting and serum was collected after centrifugation. The serum samples were stored in a $-70^{\circ} \mathrm{C}$ low-temperature freezer. Enzyme-linked immunosorbent assay (ELISA) was used to detect the levels of PGI, PGII and G-17 in the serum. The PGI, PGII and G-17 antibodies used in the enzyme immunoassay were highly specific monoclonal antibodies and were operated strictly according to the kit instructions.

Statistical analysis. The serum PGI, PGII G-17 levels and the PGR of the two groups were all expressed as mean \pm standard deviation. The PGI, PGII and G-17 levels of the two groups were analyzed using the t-test with SPSS 17.0 statistical software. The difference was statistically significant

Table 1. Distribution of demographic data.

\begin{tabular}{lccc}
\hline Group & $\mathbf{n}$ & sex $($ male/female, $\mathbf{n})$ & age $(\mathbf{x} \pm \mathbf{s}$, years $)$ \\
\hline Control group & 86 & $48 / 38$ & $52.23 \pm 8.38$ \\
Group CAG & 68 & $29 / 39$ & $53.47 \pm 9.36$ \\
\hline
\end{tabular}

$(\mathrm{p}<0.05)$. The SPSS 17.0 statistical software, logistic regression analysis and the ROC curve were used to calculate sensitivity, specificity and the best boundary value.

\section{Results}

Comparison of the serum levels of PGI, PGII, G-17 and the PGR in the two groups. The serum levels of PGI and PGR in the two groups were significantly lower than those in the control group (CAG) and the difference was significant $(\mathrm{p}<0.01)$. The PGII and G-17 levels of the CAG group were not significantly different from those of the control group ( $p>0.05$ ), as shown in Table 2.

Sensitivity, specificity and optimal threshold value of serum PGI, PGII and G-17 levels and the PGR in diagnosing CAG. Considering CAG as the case group and the normal control group as the reference, ROC curves of PGI, PGII, PGR and G-17 were plotted (Figure 1). The optimal critical values of PGI, PGII, PGR and G-17 for CAG diagnosis were 98.10 and $6.92 \mu \mathrm{g} / \mathrm{l}$, and 15.77 and 1.94 pmol/l, respectively, as shown in Table 3 .

Sensitivity, specificity and AUC of the combination of serum markers in diagnosing CAG. The sensitivity, specificity and AUC of the combination of PGI and G-17 in diagnosing CAG were $69.10 \%, 64.00 \%$ and 0.728 , respectively. The sensitivity, specificity and AUC of the combination of PGII and G-17 in diagnosing CAG were 57.40\%, $51.20 \%$ and 0.507 , respectively. The sensitivity, specificity and AUC of the combination of PGR and G-17 in diagnosing CAG were $69.10 \%, 72.10 \%$ and 0.741 , respectively (Table 4 , Figure 2).

\section{Discussion}

The results of our study showed that PGI and PGR had a relatively high accuracy in screening for CAG and predicting the risk of gastric cancer (AUCs of 0.728 and 0.726 , respectively). The high accuracy of PG in screening for CAG or GC has been reported in previous studies as well. One study [10] reported a sensitivity and specificity of PG for early screening

Table 2. Levels of serum gastric markers in different groups.

\begin{tabular}{|c|c|c|c|c|c|}
\hline Group & $\mathbf{n}$ & PGI (ng/l) & PGII (ng/l) & PGI/PGII ratio & G-17 (pmol/l) \\
\hline Control group & 86 & $126.98 \pm 41.21$ & $8.67 \pm 6.18$ & $17.09 \pm 6.27$ & $6.70 \pm 10.36$ \\
\hline Group CAG & 68 & $93.25 \pm 38.00$ & $8.16 \pm 4.16$ & $12.67 \pm 4.80$ & $5.91 \pm 9.92$ \\
\hline
\end{tabular}

Table 3. The accuracy of different serum gastric markers in diagnosing CAG.

\begin{tabular}{lcccc}
\hline Serum marker & Sensitivity (\%) & Specificity (\%) & AUC & 95\%CI \\
\hline PGI (ng/l) & 72.10 & 61.80 & $0.728 \pm 0.041$ & $0.648 \sim 0.807$ \\
PGII (ng/l) & 58.10 & 51.50 & $0.516 \pm 0.047$ & $0.423 \sim 0.608$ \\
PGI/PGII ratio & 61.60 & 77.90 & $0.726 \pm 0.041$ & $0.645 \sim 0.806$ \\
G-17 (pmol/l) & 59.30 & 55.90 & $0.556 \pm 0.048$ & $0.462 \sim 0.650$ \\
\hline
\end{tabular}



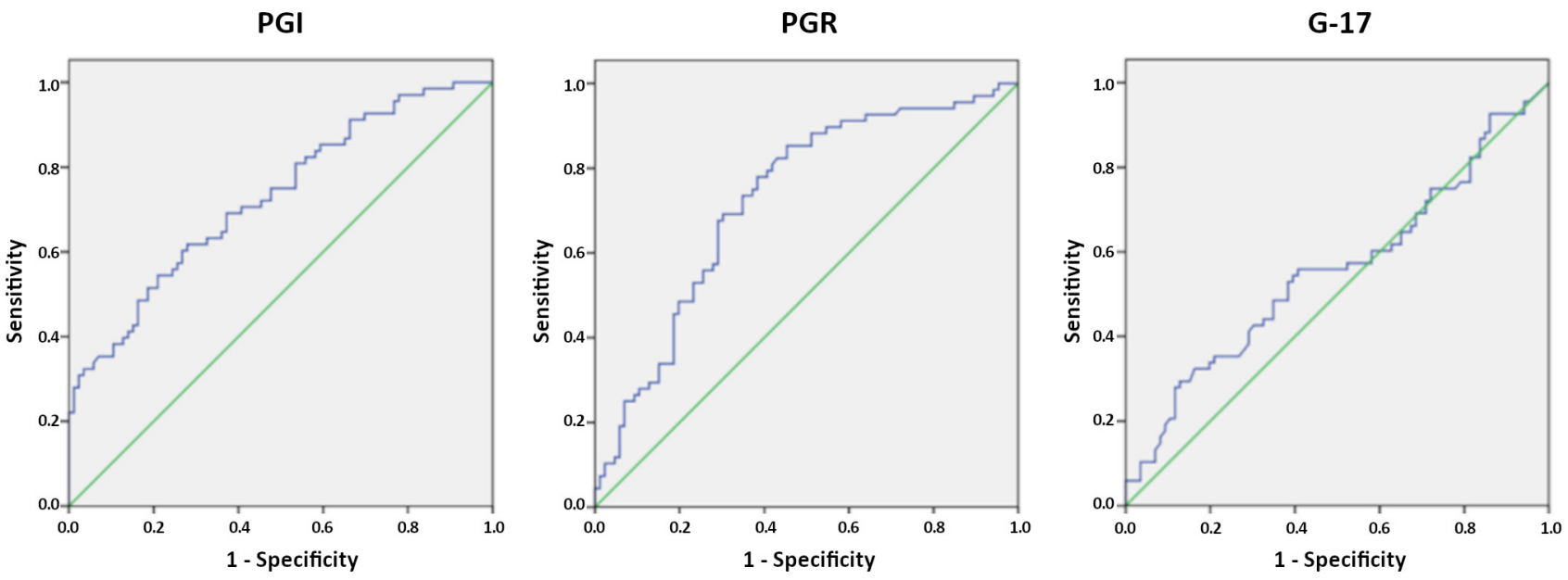

Figure 1. The ROC curve of PGI, PGR and G-17 in CAG
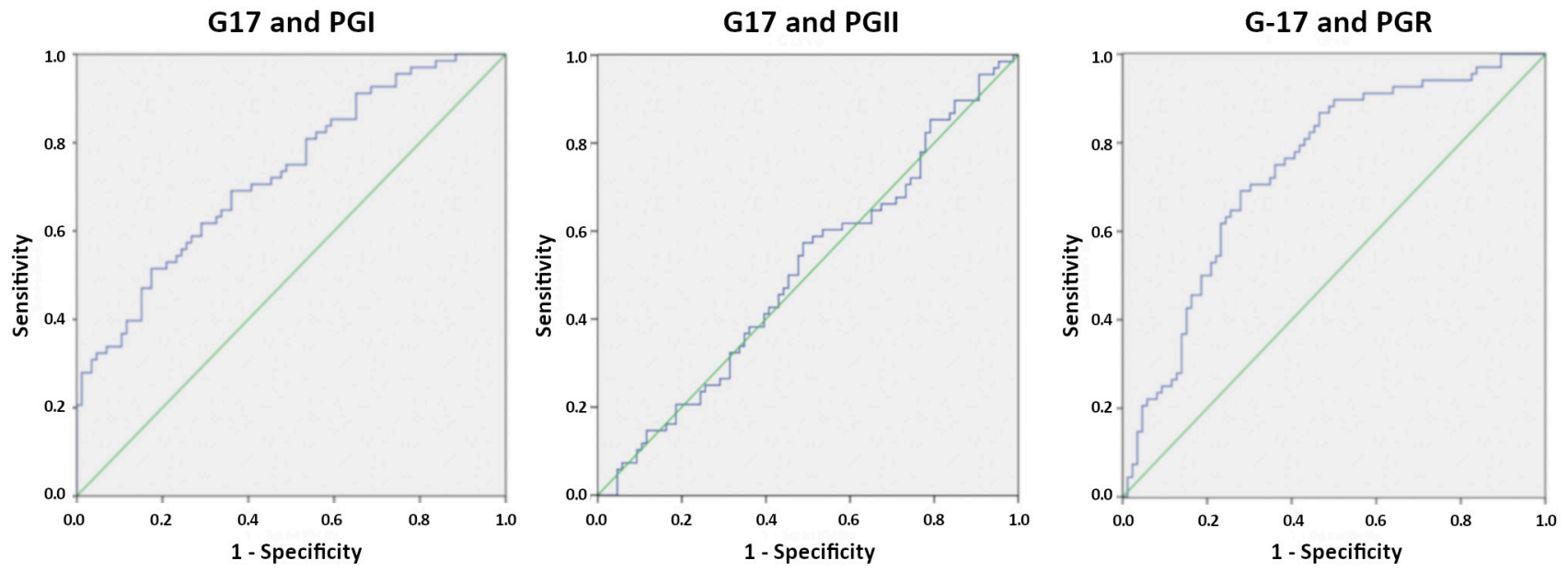

Figure 2. The ROC curve for combination of serum G-17 and PGI/PGII/PGR in diagnosing CAG

Table 4. The combination of serum G-17 with PGI, PGII and PGR in diagnosing CAG.

\begin{tabular}{lcccc}
\hline Serum marker & Sensitivity (\%) & Specificity (\%) & AUC & 95\%CI \\
\hline PGI (ng/l) and G-17 (pmol/l) & 69.10 & 64.00 & $0.728 \pm 0.040$ & $0.649 \sim 0.807$ \\
PGII (ng/l) and G-17 (pmol/l) & 57.40 & 51.20 & $0.507 \pm 0.047$ & $0.415 \sim 0.599$ \\
PGI/PGII ratio and G-17 (pmol/l) & 69.10 & 72.10 & $0.741 \pm 0.040$ & $0.663 \sim 0.820$ \\
\hline
\end{tabular}

of GC of $67 \%$ and $47 \%$, respectively. Coincidentally, another study [11] reported that the AUC of PGI and PGI combined with PGI/PGII screening for gastric atrophy (GA) were 0.78 and 0.79 , respectively. Therefore, PGI and PGI combined with PGI/PGII can be used as effective biological indicators for screening of GA and predicting the risk of gastric cancer, as well as for screening individuals in high-risk areas of gastric cancer. This can improve the diagnostic rate of gastric precancerous lesions and early gastric cancer, lead to timely treatment and a consequent improvement in the quality of patients' life. Our study showed that the AUC of G-17 screening for CAG was relatively low (about 56\%), which is consistent with the findings of Shafaghi [12], who reported an AUC of G-17 screening for GA of 59\%. However, some preliminary studies have shown that G-17 has a relatively high accuracy in screening of GA or GC. Nasrollahzadeh [11] reported that the area under the curve of G-17 for screening of GA was $77 \%$. The study by Kikushi and other studies have shown that G-17 can be used as a good biological indicator for diagnosing GA. Therefore, the validity of G-17 screening 
for predicting GA and the risk for gastric cancer remains to be further confirmed.

Our study showed that the sensitivity of the combination of gastrin G-17 and PGI for diagnosing CAG was $69.10 \%$, which was lower than using PGI alone; however, the specificity (64\%) was higher. The sensitivity of gastrin G-17 combined with PGR in diagnosing CAG was $69.10 \%$, which is higher than that of PGR alone; however, the specificity was lower than that of only PGR. Combination of serum indicators can raise the diagnostic accuracy of CAG in some respects. In the future clinical work, when the serum indicators are used to diagnose CAG, two of which can be combined flexibly to raise the accuracy. When combined detection yielded positive results, it was necessary to screen the patients carefully using gastroscopy for early diagnosis and treatment.

In conclusion, the use of serum indicators has brought convenience to the diagnosis of CAG. However, the sample size of our study is small, which is not enough to be extended to the level of screening. Therefore, it is necessary to conduct further research with a larger sample size, in order to accurately determine the sensitivity and specificity of combined detection of serum indicators.

\section{References}

[1] KIKUCHI R, ABE Y, IIJIMA K, KOIKE T, ARA N et al. Low serum levels of pepsinogen and gastrin 17 are predictive of extensive gastric atrophy with high-risk of early gastric cancer. Tohoku J Exp Med 2011, 223: 35-44. https://doi. org/10.1620/tjem.223.35

[2] VANNELLA L, LAHNER E, ANNIBALE B. Risk for gastric neoplasias in patients with chronic atrophic gastritis: A critical reappraisal. World J Gastroenterol 2012; 18: 1279-1285. https://doi.org/10.3748/wjg.v18.i12.1279

[3] KONO S. Gastric Cancer, pp 215-222. In: SR Quah, WC Cockerham (Eds.). International Encyclopedia of Public Health, Second edition, Elsevier 2017. ISBN 978-0-12803678-5.
[4] ZHENG RS, SUN KX, ZHANG SW, ZENG HM, ZOU XN et al. [Report of cancer epidemiology in China, 2015]. Zhonghua Zhong Liu Za Zhi 2019; 41: 19-28. doi: 10.3760/cma.j.i ssn.0253-3766.2019.01.005

[5] VENERITO M, NARDONE G, SELGRAD M, ROKKAS T, MALFERTHEINER P. Gastric cancer: epidemiologic and clinical aspects. Helicobacter 2014; 19 (Suppl. 1): 32-37. https://doi.org/10.1111/hel.12164

[6] ISLAMI F, SHEIKHATTARI P, REN JS, KAMANGAR F. Gastric atrophy and risk of oesophageal cancer and gastric cardia adenocarcinoma: a systematic review and meta-analysis. Ann Oncol 2011; 22: 754-760. https://doi.org/10.1093/ annonc/mdq411

[7] HOSSEINI M, AMOUEIAN S, ABANGAH G, MONTAZER $\mathrm{M}$, SOLTANI G et al. Serum gastrin-17, pepsinogen I and pepsinogen II in atrophic gastritis patients living in NorthEast of Iran. J Res Med Sci 2013; 18: 225-229.

[8] LIN JT. Screening of Gastric Cancer: Who, When, and How. Clin Gastroenterol Hepatol 2014; 12: 135-138. 10.1016/j. cgh.2013.09.064

[9] BODA T, ITO M, YOSHIHARA M, KITAMURA Y, MATSUO T et al. Advanced method for evaluation of gastric cancer risk by serum markers: determination of true low-risk subjects for gastric neoplasm. Helicobacter 2013; 19: 1-8. https://doi.org/10.1111/hel.12101

[10] LOMBA-VIANA R, DINIS-RIBEIRO M, FONSECA $\mathrm{F}$, VIEIRA AS, BENTO MJ et al. Serum pepsinogen test for early detection of gastric cancer in a European country [J]. Eur J Gastroenterol Hepatol 2012; 24: 37-41. https://doi. org/10.1097/MEG.0b013e32834d0a0a

[11] NASROLLAHZADEH D, AGHCHELI K, SOTOUDEH M, SHAKERI R, PERSSON EC et al. Accuracy and cut-off values of pepsinogens I, II and gastrin-17 for diagnosis of gastric fundic atrophy: influence of gastritis. PLoS One 2011; 6: e26957. https://doi.org/10.1371/journal.pone.0026957

[12] SHAFAGHI A, MANSOUR-GHANAEI F, JOUKAR F, SHARAFKHAH M, MESBAH A et al. Serum gastrin and the pepsinogen I/II ratio as markers for diagnosis of premalignant gastric lesions. Asian Pac J Cancer Prev 2013; 14: 3931-3936. 